\title{
A Study on Sliding Surface Pressure Measurement of Piston Ring under Reciprocating Condition Using Thin-Film Sensor
}

\author{
Kazuya Mochizuki $i^{1)^{*}}$, Yosuke Watanabe $^{1)}$, Michiyasu Owashi ${ }^{2)}$ and Yuji Mihara ${ }^{2)}$ \\ ${ }^{1)}$ Graduate School of Engineering, Tokyo City University, 1-28-1 Tamazutsumi, Setagaya-ku, Tokyo 158-8557, Japan \\ ${ }^{2)}$ Department of Mechanical Engineering, Tokyo City University, 1-28-1 Tamazutsumi, Setagaya-ku, Tokyo 158-8557, Japan \\ *Corresponding author: Kazuya Mochizuki (g1691101@tcu.ac.jp)
}

Manuscript received 17 June 2019; accepted 12 September 2019; published 31 October 2019

\begin{abstract}
In the tribology of mechanical elements using lubricating oil, verification of the dynamic surface pressure distribution during sliding operation is necessary in order to elucidate the sliding condition. The same also applies to the piston rings which are the sliding part of compression reciprocating device. Although some verification case in static condition or limited sliding area has been studied, there are many unknown aspects in this field. The thin-film sensor, since it has small influence on the shape of the sliding surface, is widely used as a measurement method of the sliding surface pressure between two different surfaces, however this method has never been applied to the piston ring in the past. In this study, various film deposition methods on the piston ring of the thin-film sensor were examined, and the thin-film pressure sensor with a total thickness of $4.6 \mu \mathrm{m}$ was successfully formed on the sliding surface by sputtering. The surface pressure distribution of the sliding part was measured using the reciprocating sliding tester by this thin-film pressure sensor, and the measurement values were in good agreement with the mixed lubrication model between the piston ring and the cylinder liner.
\end{abstract}

\section{Keywords}

piston ring, surface pressure, thin-film sensor, reciprocating

\section{Introduction}

In the tribology of mechanical elements using lubricating oil, the increase or decrease of the surface pressure, and the surface pressure distribution generated on sliding surfaces greatly affect the friction force and the wear resistance. Therefore, it is very important to correctly understand the dynamic surface pressure distribution during sliding condition. The same applies to the piston ring, which is a seal part of a reciprocating device that requires a fluid compression stroke. The example of the surface pressure measurement performed in the past, Brown, S. R. et al. [1] measured oil-film pressure between piston ring and cylinder bore using a piezo quartz sensor in sliding condition. And, it was confirmed that the variation of oil-film pressure in several hundred $\mathrm{kPa}$ was generated. Also, Hitosugi, H. et al. [2] and Cheng, C. et al. [3] measured the static surface pressure distribution using rig test device. However, these results were limited to the specific location in the sliding area and the measurement results in the static condition, there are many unknown aspects in this field. In addition, as piston ring models for theoretical calculation, Rohde [4] proposed a mixed lubrication model that was a combination of the average Reynolds model proposed by Patir \& Cheng [5] and the stochastic contact theory proposed by Greenwood \& Tripp [6].
This method had been used by subsequent researchers widely. In recent years, various boundary conditions for this model, the effect of temperature distribution in the oil-film and the surface temperature of parts have been continuously studied[7, 8]. In addition, conformability to cylinder bore $[3,9,10]$, consideration of detailed surface properties including roughness with the development of surface texturing technology have been studied $[11,12]$. However, as described above, since there is no surface pressure measurement method in a dynamic environment where the actual piston ring slides in the cylinder bore, the correlation with the actual phenomenon has not been clarified.

For the sliding surface pressure measurement between two different surfaces, the thin-film sensor is widely used, as it has small influence on the shape of the sliding surface $[13,14]$. However, this method has never been applied to the surface pressure distribution measurement between the piston ring and the cylinder bore in the past.

Authors formed the thin-film pressure sensor using DLC (Diamond Like Carbon) by sputtering method and PCVD (Plasma Chemical Vapor Deposition) method on the sliding surface of the piston ring, and performed the instantaneous pressure measurement between the piston ring and the cylinder bore. In addition, in order to measure pressure distribution using a reciprocating sliding tester consisting of the piston, 
the piston ring, and the cylinder, the low noise measurement system was developed to expand the measurable range to pressures below $1 \mathrm{MPa}$. Furthermore, the validity of the analysis was investigated by verifying the measurement results and theoretical calculation results of the surface pressure.

\section{Thin-film pressure sensor}

\subsection{Measurement principle}

The resistant value of $\mathrm{Cu}-\mathrm{Mn}-\mathrm{Ni}$ alloy, the pressure sensing material, changes in conjunction with the pressure. This resistance change $\Delta R$ is converted to the voltage change $\Delta e$ by the Wheatstone bridge circuit shown in Fig. 1, and then the pressure measurement will be possible. The variable resistor is installed close to the sensor in order to adjust the individual difference or the valance of the sensor. The resistance change $\Delta R$ is approximated by Eqs. (1) and (2).

$$
\begin{aligned}
& \Delta e=\frac{1}{4} \cdot E \cdot \frac{\Delta R}{R} \\
& \frac{\Delta R}{R}=\alpha_{P} \cdot \Delta P \quad\left(\alpha_{P}: \text { Pressure sensitivity }\right)
\end{aligned}
$$

\subsection{Application of the thin-film pressure sensor for the piston ring}

Figure 2 and Table 1 shows the shape and specifications of the piston ring tested in this study. Figure 3 shows the shape of the sensor formed on the sliding face of the piston ring. The thin-film sensor is composed of the sensing part and the leading part, made of $\mathrm{Cu}-\mathrm{Mn}-\mathrm{Ni}$ alloy film. In the case of the piston ring, the sensing part was placed on the vertex position. Figure 4 shows the structure and the cross-sectional view of the thin-film pressure sensor. Layers of the Insulation film (2), the Sensing and leading films (3), as well as the DLC protection film (4) are deposited on the Substrate (1) (= piston ring surface) in a sequential order. The material and the thickness of each film

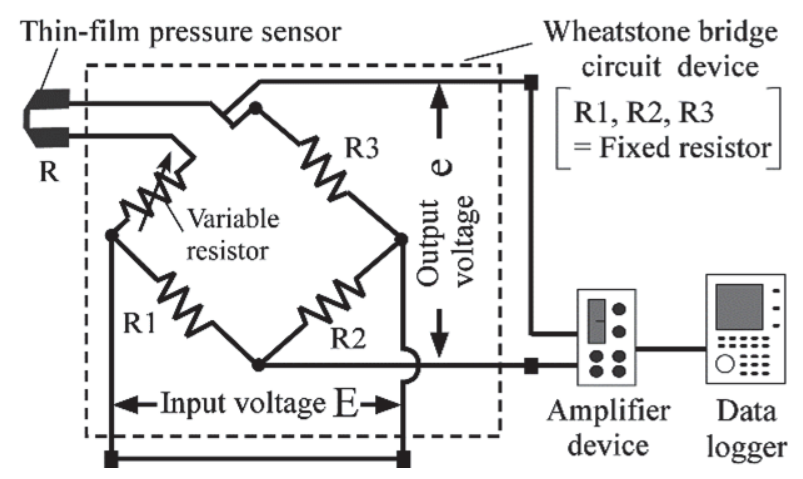

Fig. 1 Measurement system (Wheatstone bridge circuit)

Forming point of thin-film pressure sensor
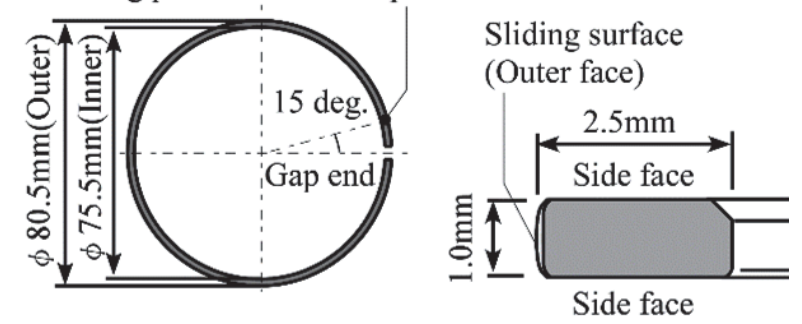

Fig. 2 Shape of the piston ring

Table 1 Specification of the piston ring

\begin{tabular}{|c|c|}
\hline Type & Compression ring (Top ring) \\
\hline Cross section & Internal bevel \\
\hline Outer face profile & Barrel face \\
\hline Material & Steel \\
\hline Tension & $3.7(\mathrm{~N})$ \\
\hline
\end{tabular}
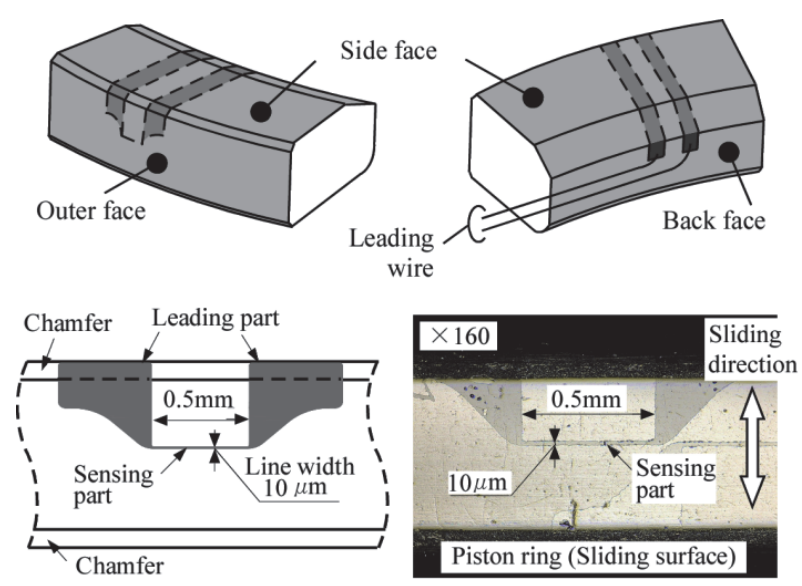

Fig. 3 Shape of the thin-film pressure sensor

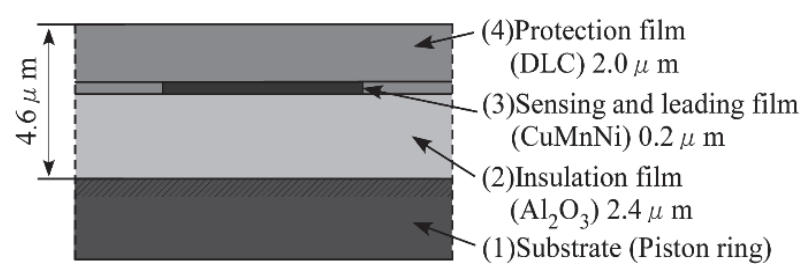

Fig. 4 Cross section of the sensor film

are as shown in Fig. 4, and the total thickness of the formed film is $4.6 \mu \mathrm{m}$. In order to prevent the shape of the sensor part from changing, a $4.6 \mu \mathrm{m}$ thick film was formed all around the ring, and a thin-film pressure sensor was successfully formed without changing the basic shape and tension of the ring.

\subsection{Outline of the measurement system}

In this research, the following improvements were made to the measurement system in order to realize high-precision measurement in the low pressure region [15, 16]. Figure 5 shows the improvement of the Wheatstone bridge circuit device. In the Wheatstone bridge circuit, increase of the input voltage $\mathrm{E}$

Thin-film pressure sensor $\mathrm{R}=150 \sim 200 \Omega$

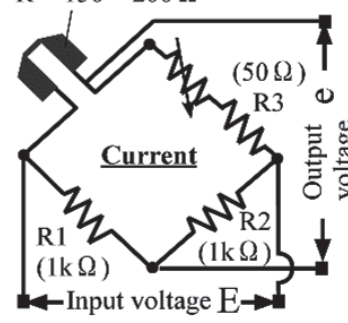

Fig. 5 Improvement of the Wheatstone bridge circuit structure 
leads to the increase of the output voltage, which improves S/ $\mathrm{N}$ ratio (the ratio of the output signal voltage against the noise voltage) [17]. However, at the same time, increase of the input voltage leads to the increase of the Joule heat, which causes the zero-point drift (collapse of equilibrium). Therefore, the layout, resistance value, and temperature resistance coefficient of each component of the Wheatstone bridge circuit were improved. Next, for the purpose of further reduction of the noise, the improvement of the strain amplifier was conducted. The simplified diagram of the structure of amplifier device is as shown in Fig. 6. In order to achieve both the internal gain increase and the noise reduction, filtering reinforcement at pre-amplifier, gain adjustment at buffer circuit, and coefficient adjustment at $\mathrm{A} / \mathrm{D}$ converter were applied.

In order to verify the effect of combining these

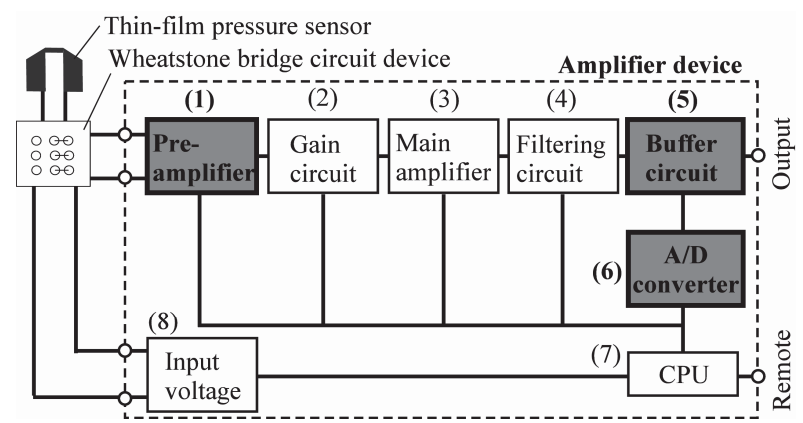

Fig. 6 Improvement of the amplifier device

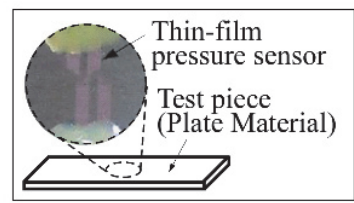

(3)Thin-film pressure sensor

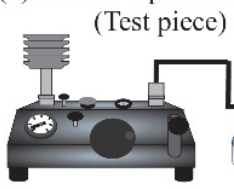

(1)Pressure gauge

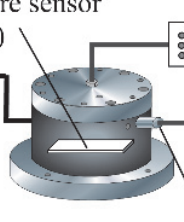

(2)Pressure vessel
(4)Wheatstone bridge circuit device

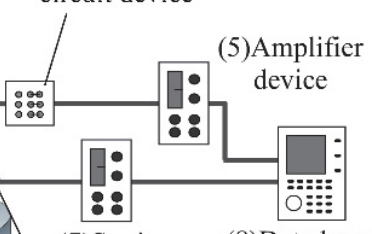

(7) Strain amplifier

(8)Data logger

(6)Pressure transducer

(Standard for pressure)
Fig. 7 The pressure tester of the thin-film pressure sensor

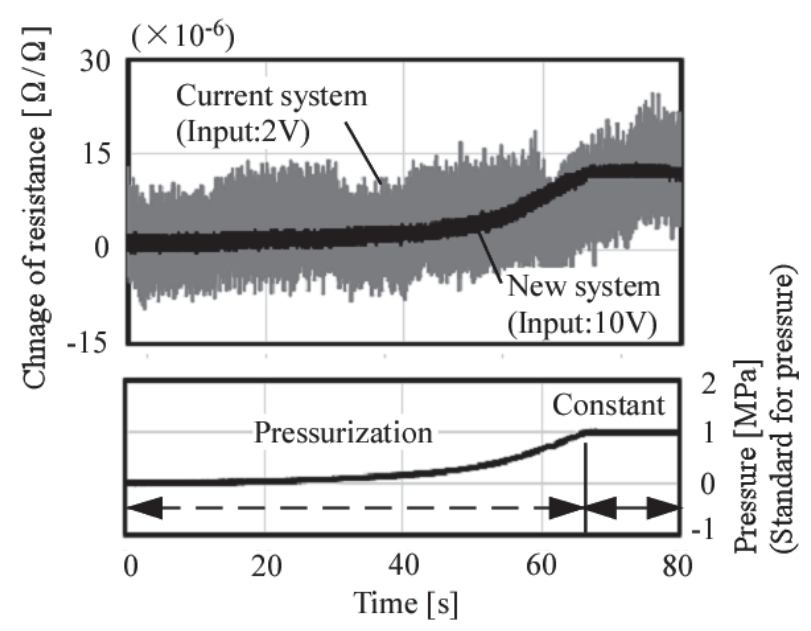

Fig. 8 Pressure test result of the test piece improvements, the result of the pressure test conducted on the thin-film pressure sensor, which is formed on the test piece, as well as on the improved system, by using the test machine in Fig. 7, is shown in Fig. 8. Here, the measurement of the improved system was conducted under the input voltage 10 $\mathrm{V}$. The measurement system after the improvement, including the effect by the increased input voltage compared to the current system, has resulted in its noise width reduced greatly. Furthermore, Fig. 9 shows the S/N ratio derived by the thin-film pressure sensor output voltage at every $0.1 \mathrm{MPa}$ pressurization, as well as the noise voltage. The $\mathrm{S} / \mathrm{N}$ ratio is shown in Eq. (3), and this equation includes the noise voltage (peak-to-peak) Vn and the output signal voltage $V_{s}$.

$$
\frac{S}{N}=20 \cdot \log _{10} \frac{V S}{V n}
$$

A 42.9 to $62.9 \mathrm{~dB}$ improvement (87.1 to $149.4 \%$ ) was seen in the whole region under $1 \mathrm{MPa}$ compared to the current system, and the region of $\mathrm{S} / \mathrm{N}$ ratio $>0$ was drastically increased. Furthermore, examination results of the frequency characteristics are indicated in Figs. 10 and 11. In this study,

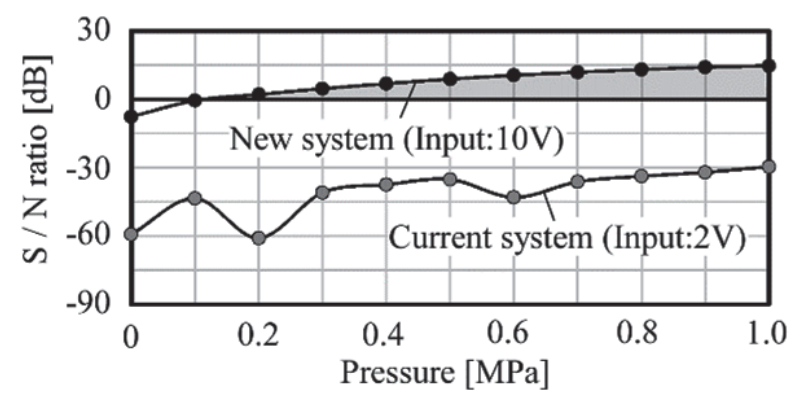

Fig. $9 \mathrm{~S} / \mathrm{N}$ ratio of the current measurement system and the new measurement system

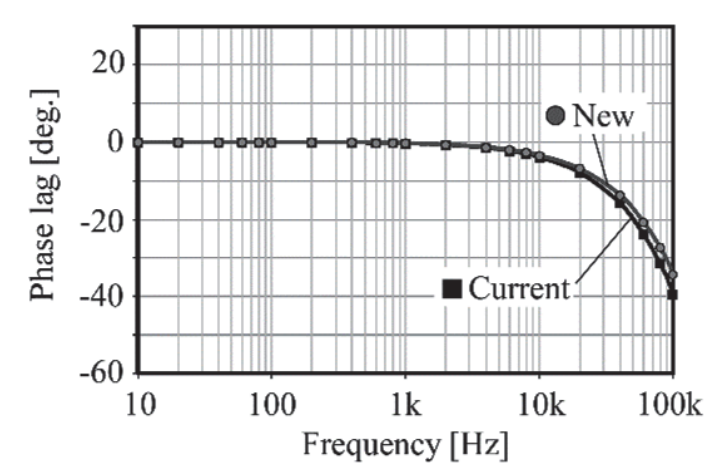

Fig. 10 Test result of phase lag

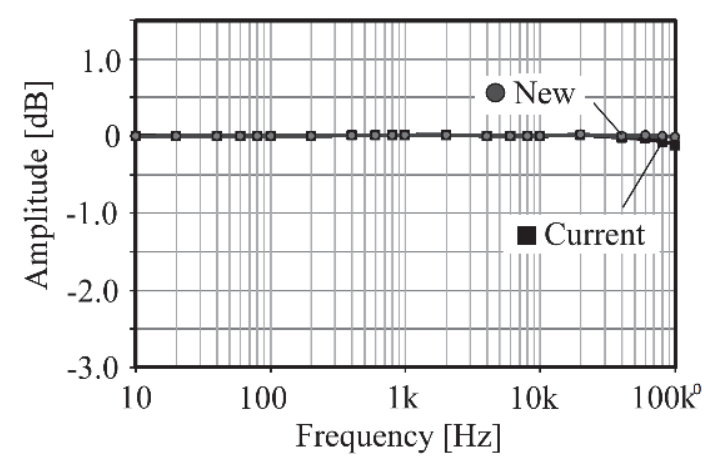

Fig. 11 Test result of amplitude 
examination was performed with a maximum condition of 1000 $\mathrm{rpm}(16.7 \mathrm{~Hz})$ in the reciprocating sliding test with a pistoncrank mechanism. In the new measurement system, the phase delay was improved up to 5 degree $(13.2 \%)$ in the region below $100 \mathrm{kHz}$. In addition, the amplitude was improved up to 0.12 $\mathrm{dB}(95.1 \%)$ in the same frequency range.

\section{Calibration of the thin-film pressure sensor}

\subsection{Pressure sensitivity}

Figure 12 shows the calibration system of the sensor pressure sensitivity. The test oil is supplied from the Pressure gauge (1) and the thin-film pressure sensor (3), which is formed on the piston ring, is pressurized in the Pressure vessel (2). At this point, the output signal which passes through the Wheatstone bridge device (4) and the Amplifier device (5) is recorded by the Data logger (8). Here, the output (pressure sensitivity) per unit pressure is obtained from the relation between the pressure indicated value and the sensor output voltage value by installing the pressure transducer which is calibrated in advance in the vicinity of the sensor. Figure 13 shows a representative example of the pressure calibration result. The output voltage was proportional to the pressure change, which shows high linearity from the correlation coefficient $\left(R^{2}=0.9995\right)$. In addition, $12.08 \mu \Omega / \Omega / \mathrm{MPa}$ was derived as the pressure sensitivity $\alpha_{p}$. Furthermore, when comparing the output value widths of the current measurement system and the new measurement system, it was clear that the measurement value width were significantly improved.

\subsection{Temperature sensitivity}

Due to temperature changes when the piston ring slides

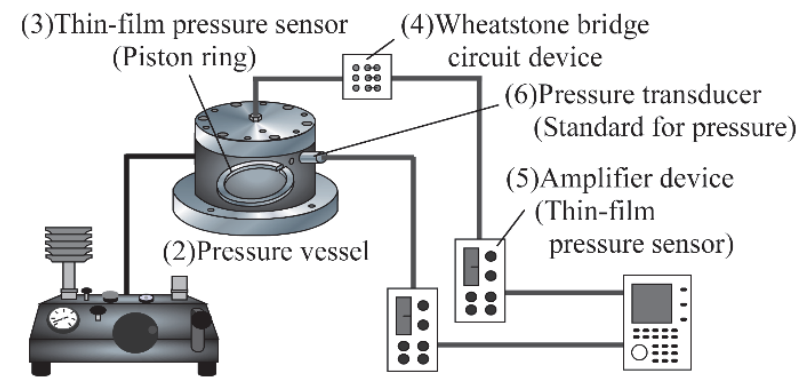

$\begin{array}{lll}\text { (1)Pressure gauge (7)Strain amplifier } & \text { (8)Data logger }\end{array}$

Fig. 12 Calibration system of pressure sensitivity

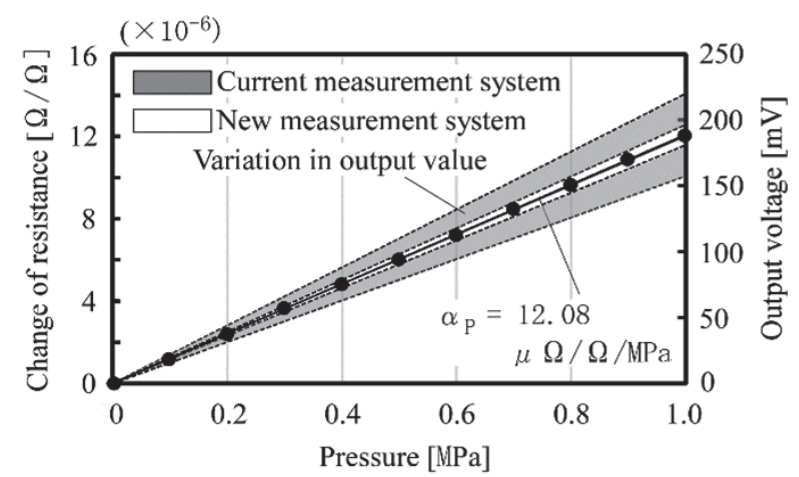

Fig. 13 Calibration result of pressure sensitivity on the cylinder liner, a pressure measurement error may occur depending on the temperature sensitivity of the sensor attached to the piston ring. Therefore, the temperature sensitivity of the sensor was examined using the calibration system of the temperature sensitivity shown in Fig. 14. The thin-film pressure sensor formed on the piston ring (2) is heated in the Program heater (1), and the output signal through the Wheatstone bridge circuit device (3) and the Amplifier device (4) is recorded by the Data logger. As a result, as shown in Fig. 15, the temperature sensitivity of the sensor was obtained $\alpha_{T}=4.84 \mu \Omega / \Omega / \mathrm{MPa}$. From this $\alpha_{T}$ and the pressure sensitivity $\alpha_{P}=12.08 \mu \Omega / \Omega / \mathrm{MPa}$, the pressure error $\alpha_{T} / \alpha_{P}$ was estimated $0.40 \mathrm{MPa} /{ }^{\circ} \mathrm{C}$.

\section{Reciprocating sliding test}

\subsection{Test machine}

The overview of the reciprocating sliding test device is shown in Fig. 16. The crosshead structure suppresses secondary piston movement, so only vertical movement is possible. The upper part of the cylinder is open to the atmosphere, so the air compression does not occur. The lubricating oil (2) is supplied from the temperature controlled tank and dripped from the upper position of the cylinder. Due to the piston ring installed upside down, the top stop position of the piston ring is Bottom Dead Center (BDC), and the bottom stop position is Top Dead Center (TDC). For temperature measurement of the sliding surface, (5) a thermocouple installed on the central wall surface of the stroke was used as shown in Fig. 17. At this time, GF-5 SN $0 \mathrm{~W}-20$ was used as the test oil. The leading wire of the thin-film pressure sensor formed on the piston ring was led out of the test machine using the linkage device (6), and was connected to the data logger through the measurement system.

\section{(2)Thin-film pressure sensor (Piston ring)}

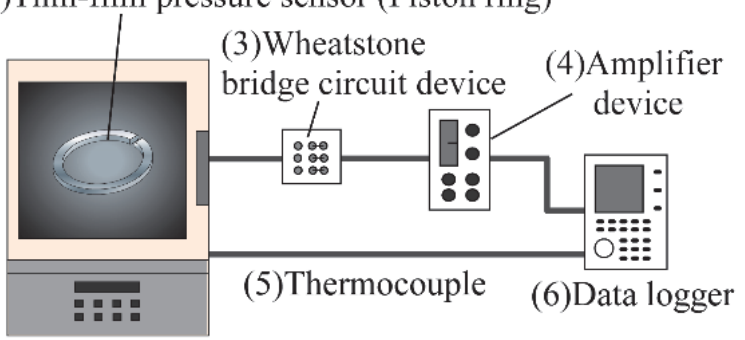

(1)Program heater

Fig. 14 Calibration system of temperature sensitivity

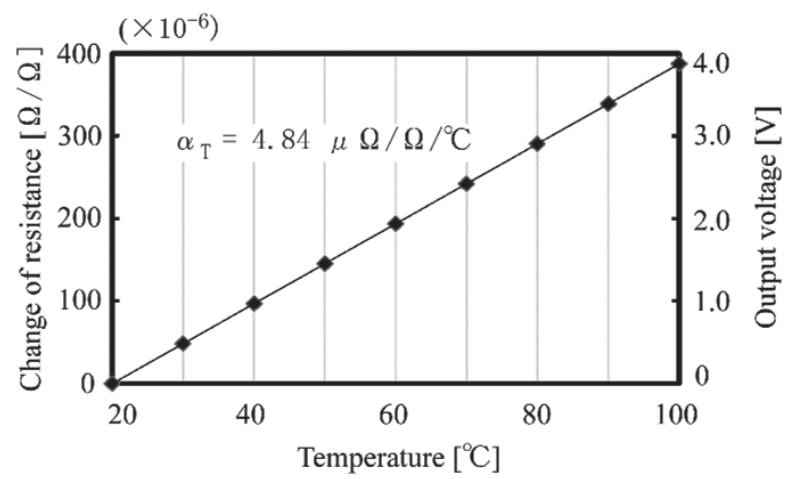

Fig. 15 Calibration result of temperature sensitivity 


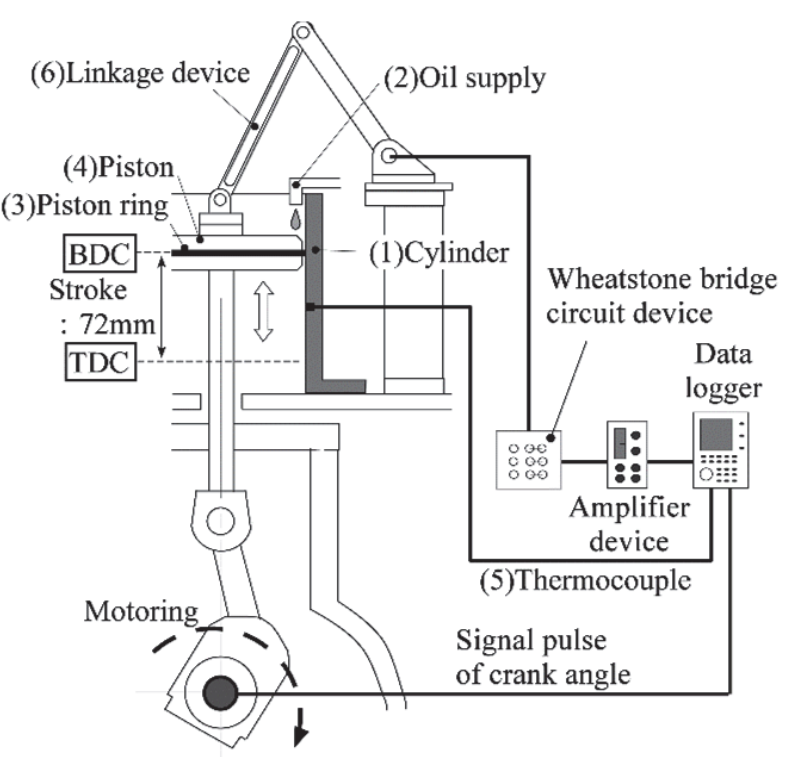

Fig. 16 Schematic of the reciprocating sliding tester

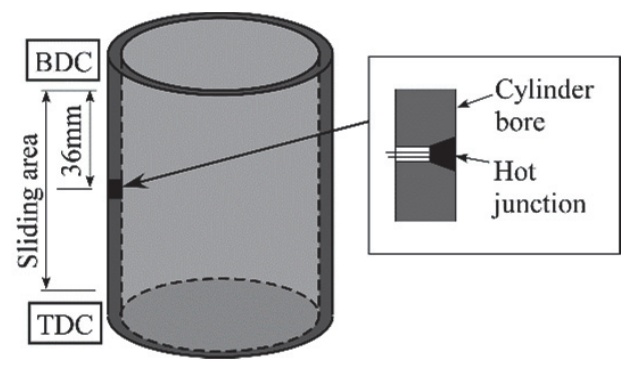

Fig. 17 Schematic of the thermocouple installing

4.2 Determination of the zero-point in the sliding surface pressure measurement results

In order to clearly determine of zero-point in the measurement value by the thin-film pressure sensor, the measurement using a piezo quartz sensor was performed with reference to the measurement method of Brown, S. R. et al. [1]. As shown in Fig. 18, through holes were drilled and the measurement probe and piezo quartz sensor were placed. After that, preload was applied by tightening the lock nut, and the end face of the measurement probe and the cylinder bore were co-honed. The zero-point of a measurement value of the thin-film pressure sensor was determined by making the surface pressure measurement value at the sensor installation position and the thin-film pressure sensor measurement value at the same position identical. The measurement results of the

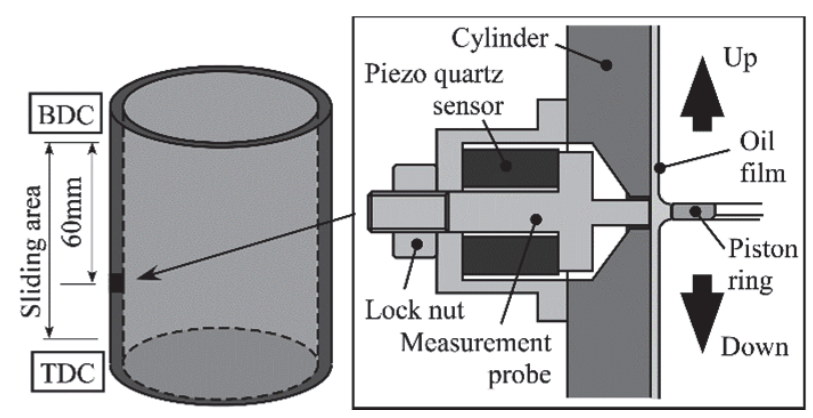

Fig. 18 Schematic of the piezo quartz sensor implanting

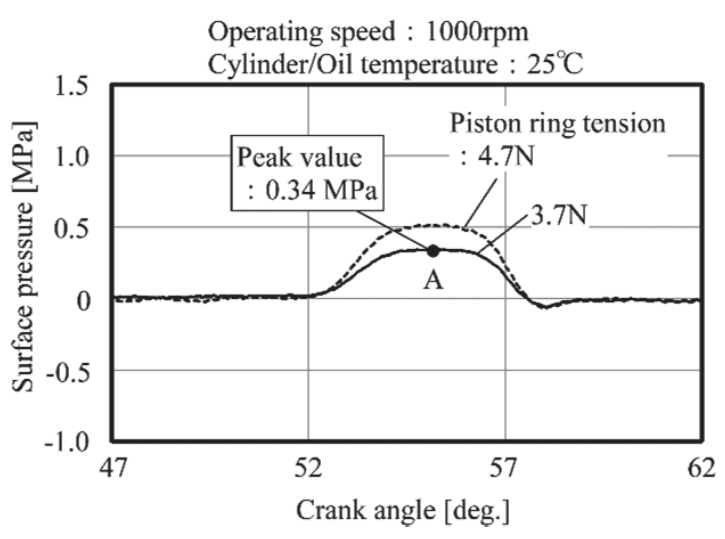

Fig. 19 Measurement result of the piezo quartz sensor test

piezo quartz sensor are shown in Fig. 19. When the piston ring passes over the measurement probe (crank angle of 53.5 to 58.5 degree), A surface pressure of up to $0.34 \mathrm{MPa}$ (the piston ring tension of $3.7 \mathrm{~N}$ ) was generated at point $\mathrm{A}$. In addition, the surface pressure value increased with the increase of the piston ring tension value.

\subsection{Theoretical calculation model and conditions}

The theoretical calculation was performed using EXCITE PISTON \& RINGS [18] manufactured by AVL GmbH. For simulating the sliding condition of the test device shown in Fig. 16, the secondary piston movement was eliminated, and the condition that one TOP ring to reciprocate in the cylinder was set as shown in Figs. 20 and 21. At this time, only the TOP ring was used as the compression ring, the Oil Control ring

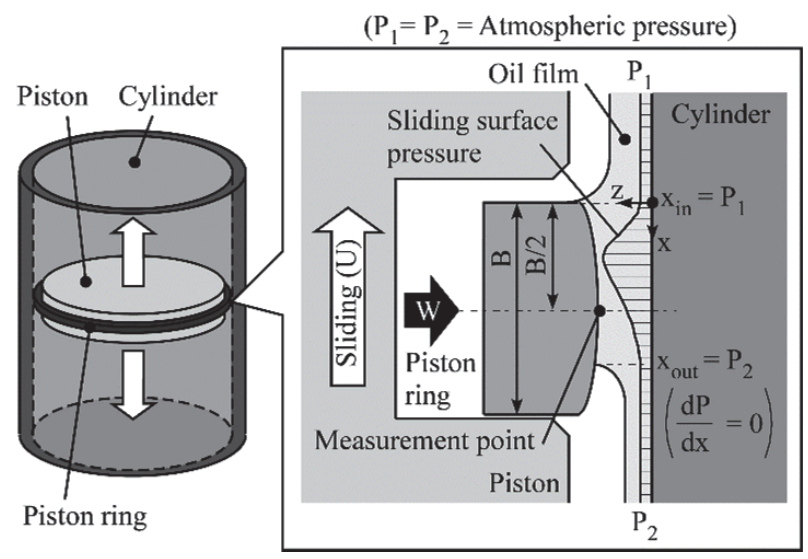

(a) Boundary condition

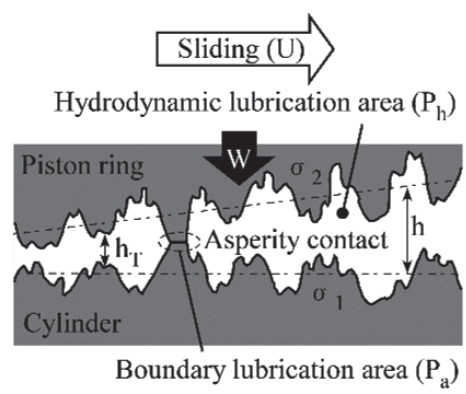

(b) Mixed lubrication model

Fig. 20 Theoretical calculation model 


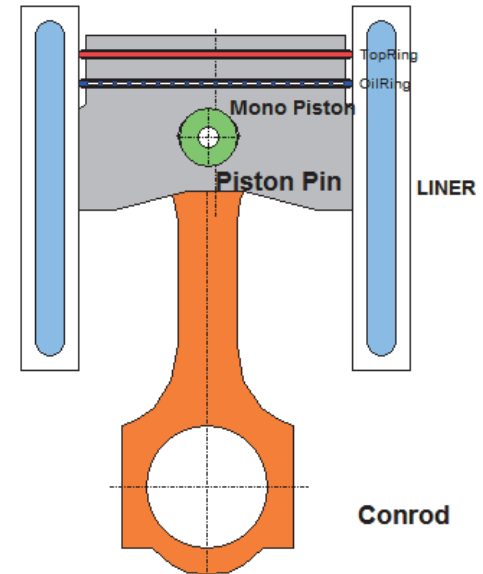

Fig. 21 GUI of EXCITE PISTON \& RINGS

tension was set to zero for convenience, and the pressure in the surrounding space was equivalent to the atmospheric pressure. In the hydrodynamic lubrication evaluation, the average Reynolds equation by Patir \& Cheng [5] in consideration of the surface roughness shown in Eqs. (4) to (7), and in the asperity contact evaluation, the roughness contact model by Greenwood \& Tripp [6] shown in Eqs. (8) and (9) was used. These are proposed as a mixed lubrication model by Rohde [4]. The reason for using this model is that in the reciprocating motion by the piston-crank mechanism, the piston and cylinder slide through a very thin oil-film. However, when the reciprocating direction is switched, the piston speed becomes 0 , and asperity contact occurs when the oil-film is not sufficiently formed. Therefore, as shown in Fig. 20 (b), the lubrication conditions require a model that can consider both fluid lubrication and asperity contact. Although some assumptions are required, the mixed lubrication model by Rohde was adopted in this study. The characteristics are described below;

- Due to the probabilistic handling of effects of actual surface roughness with random characteristic, handling of average effect of surface roughness is possible.

- Ideal in determining average pressure distribution and oilfilm thickness in contact region

- From the above, the mixed lubrication model proposed by Rohde (including the average flow model by Patir \& Cheng and the contact model by Greenwood \& Tripp) have been applied to many piston ring lubrication models in the past.

- It thought that these models are useful as a first stage in the validation of the measurement results by the newly developed measurement method, including the past study's knowledge.

As shown in Fig. 20(a), the Reynolds boundary conditions were used in this study. At the front end of the piston ring, the oil flows in at pressure $P=P_{1}$ (atmospheric pressure). Next, $P$ becomes $P_{2}$ (atmospheric pressure) at the point where the pressure gradient becomes $0(d P / d x=0)$, and the oil flows out. In addition, the calculation model assumes the following conditions;

- Piston rings are extremely long in the circumferential direction with respect to the sliding direction, and can be treated as a case of two-dimensional fluid lubrication.

- Lubricant is a Newtonian fluid

- Fluid viscosity is constant

- Fluid temperature is constant

- Flow is a laminar flow
- Inertial force of fluid is ignored

- Fluid is incompressible

- Fluid density is constant

- Oil-film pressure is uniform in film thickness direction

- Fluid does not slide at boundary with wall surface

- Wall surface is a rigid body (no deformation)

At first, Eq. (4) is integrated twice for $\mathrm{x}$, and the average oilfilm pressure $\bar{P}$ is obtained using Eqs. (5)-(7) and the boundary conditions described above. The average oil-film pressure $\bar{P}$ is further integrated from the oil-film start position $x_{i n}$ to the oil-film end position $x_{\text {out }}$. So, The load $P_{h}$ due to the oil-film pressure is obtained. In this study, the roughness orientation function used by Patir \& Cheng was used. At this time, the roughness orientation function specified by Patir \& Cheng was treated as the case of isotropic roughness $(\gamma=1)$. Next, Eqs. (8) (and (9)) is further integrated from the oil-film start position $x_{i n}$ to the oil-film end position $x_{\text {out }}$. So, the load $P_{a}$ due to asperity contact is obtained. Since the sum of the load $P_{h}$ and the load $P_{a}$ is balanced with the radial load $W$ (by the piston ring tension), Eq. (10) is established. From this balance equation, the change in the oil-film thickness $\Delta h$ is obtained as a first-order ordinary differential equation including each time interval $\Delta t$. Finally, the initial oil-film thickness is set, and the cycle calculation for this first-order ordinary differential equation is executed until it is stabilized by the finite difference method. The actual shape shown in Fig. 22 was taken as point sequence data for the piston ring sliding shape.

$$
\begin{aligned}
& \frac{\partial}{\partial x}\left(\phi_{x} \frac{h^{3}}{12 \eta} \frac{\partial \bar{p}}{\partial x}\right)=\frac{U}{2} \frac{\partial \bar{h}_{r}}{\partial x}+\frac{U \sigma}{2} \frac{\partial \phi_{s}}{\partial x}+\frac{\partial \bar{h}_{T}}{\partial t} \\
& \phi_{x}= \begin{cases}1-C \exp \left(-\gamma \frac{h}{\sigma}\right) & , \gamma \leq 1 \\
1+C\left(\frac{h}{\sigma}\right)^{-\gamma} & , \gamma>1\end{cases} \\
& \phi_{s}=\left(\frac{\sigma_{1}}{\sigma}\right)^{2} \Phi_{s}\left(h, \gamma_{1}\right)-\left(\frac{\sigma_{2}}{\sigma}\right)^{2} \Phi_{s}\left(h, \gamma_{2}\right) \\
& \Phi_{s}(h, \gamma)= \begin{cases}A_{1}\left(\frac{h}{\sigma}\right)^{\alpha_{1}} \exp \left(-\alpha_{2} \frac{h}{\sigma}+\alpha_{3} \frac{h}{\sigma}\right) & , \frac{h}{\sigma} \leq 5 \\
A_{2} \exp \left(-0.25 \frac{h}{\sigma}\right) & , \frac{h}{\sigma}>5\end{cases} \\
& P_{a}=\frac{16 \sqrt{2}}{15} \pi\left(\sigma_{s} \beta \eta\right)^{2} E^{*} \frac{\sqrt{\sigma}}{\beta} \int_{x_{\text {in }}}^{x_{\text {out }}} F_{5 / 2}\left(\frac{h}{\sigma}\right) d x \\
& F_{5 / 2} \begin{cases}4.408610^{-5}\left(4-\frac{h}{\sigma}\right)^{6.804} & , \frac{h}{\sigma}<4 \\
0 & , \frac{h}{\sigma} \geq 4\end{cases} \\
& W=P_{h}+P_{a}
\end{aligned}
$$

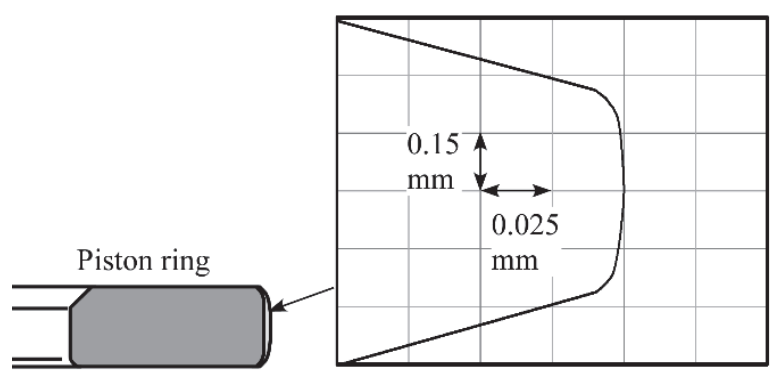

Fig. 22 Actual profile of the sliding surface 
$h \quad$ : Nominal oil film thickness

$\bar{h}_{T}$ : Average gap

$h_{T}:$ Local gap

$\bar{p} \quad$ : Mean hydrodynamic pressure

$\sigma \quad$ : Composite surface roughness

$$
\sigma=\sqrt{\left(\sigma_{1}^{2}+\sigma_{2}^{2}\right)}
$$

$\sigma_{1}$ : Surface roughness (Cylinder)

$\sigma_{2}:$ : Surface roughness (Piston ring)

$U$ : Sliding velocity

$\mu$ : Absolute viscosity

$\phi_{x}:$ Pressure flow factor

$\phi_{\mathrm{s}}$ : Shear flow factor

$\gamma, \gamma_{1}, \gamma_{2}:$ Roughness orientation

C, $r, A_{1}, A_{2}, \alpha_{1}, \alpha_{2}, \alpha_{3}$ : Constant value (determinined by $\gamma$ [5])

$p_{h} \quad$ : Load by hydrodynamic oil film pressure

$p_{a}:$ Load by asperity contact pressure

$\beta \quad$ : Peak radius at asperity summit

$\eta$ : Surface density of asperity peaks on each surface

$E^{*}$ : Composite Young's modulus in contact area

$F_{52}$ : Contact load by shape factor

$\mathrm{W}$ : load by piston ring tension

4.4 Test results

The measurement results and theoretical calculation results of the surface pressure at room temperature $\left(25^{\circ} \mathrm{C}\right)$ are shown in Fig. 23. At this time, the temperature of parts and the temperature of supplied oil were controlled to room temperature $\left(25^{\circ} \mathrm{C}\right)$. As shown in Section 4.2 (Determination of the zero-point in the sliding surface pressure measurement results), the zero-point of a measurement value was determined by applying the pressure value of the thin-film pressure sensor at the point B to $0.34 \mathrm{MPa}$ (in case of $1000 \mathrm{rpm}$ ). Since a rapid change of the waveform did not occurred near this crank angle, the zero-point was determined at this crank angle. As shown in Fig. 23, an obvious rise of the surface pressure at TDC and BDC was observed, and the behavior of the measurement waveform and the calculation waveform matched well at both (a) $500 \mathrm{rpm}$ and (b) $1000 \mathrm{rpm}$. Next, the pressure rise (Section (i ) in Fig. 23) at the TDC position that showed the most abrupt behavior was focused, and the peak values were compared. The enlarged waveform of Section ( $i$ ) at each operating speed is shown in Fig. 24(a). Here, it was confirmed that the occurrence timing of the peak position was a difference of less than 1 degree $(0.13$ to 0.85 degree). In addition, measurement and calculation values show a peak behind 0 degree (TDC). Due to the dynamic effect of the oil-film formed between the piston ring and the cylinder bore, the minimum value of the oil-film thickness occurs with a slight delay from the TDC and BDC. So, the peak surface pressure value also occurs with a slight delay [19]. The comparison result of the pressure peak value in each waveform is shown in Fig. 24(b). First, comparing the change with the operating speed, the calculation value decreases by $-0.10 \mathrm{MPa}(-15.9 \%)$ and the measurement value decreases by $-0.12 \mathrm{MPa}(-18.3 \%)$, resulting in almost the same change. In addition, focusing on the difference between the calculation value and the measurement value, the difference was -0.04 to $-0.02 \mathrm{MPa}(-6.7$ to $-3.9 \%)$, and almost the same value regardless of the operating speed.

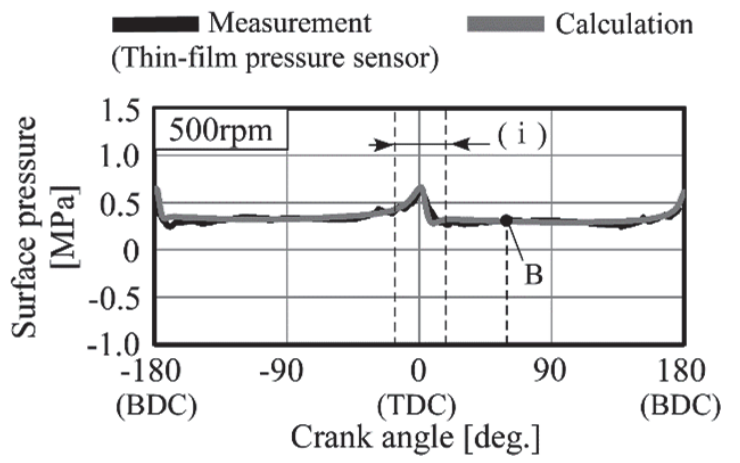

(a) $500 \mathrm{rpm}$

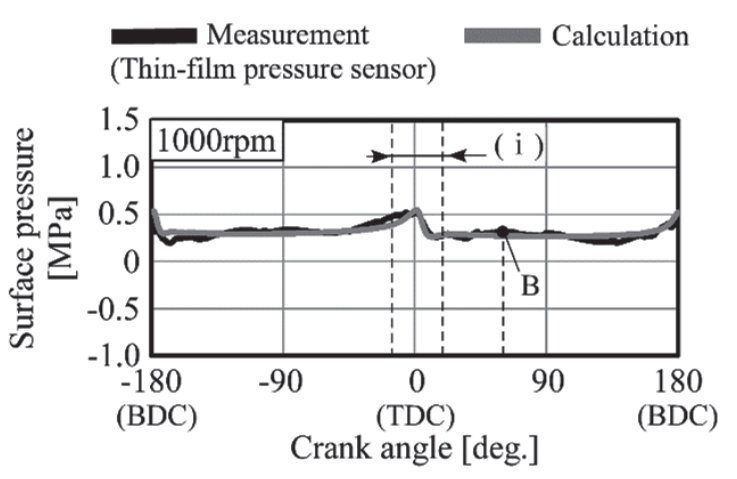

(b)1000rpm

Fig. 23 Measurement result and theoretical calculation result of the reciprocating sliding test

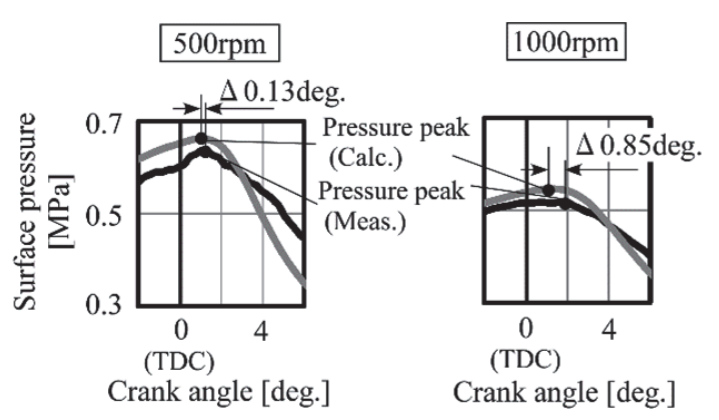

(a) Waveform at section ( i ) of Fig. 23

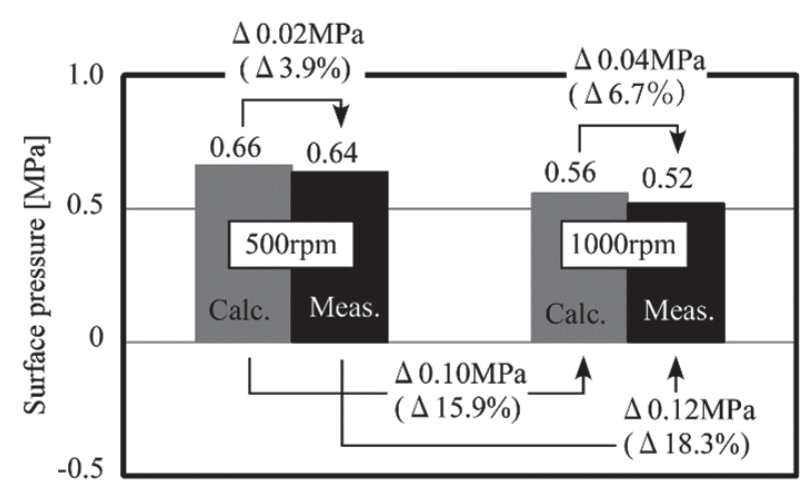

(b) Peak value at section ( i ) of Fig. 23

Fig. 24 Comparison in waveform and peak value at section (i ) of Fig. 23 
4.5 Examination of pressure measurement error due to temperature fluctuation

The possibility that the temperature sensitivity of thinfilm pressure sensors (see Section 3.2) gives surface pressure measurement values was considered. Figure 25 shows the result of measuring the temperature fluctuation near the liner surface during the surface pressure measurement test of Fig. 23. The wall temperature gradually increased from the start of operation, and the wall temperature became almost constant after 40 seconds ( $500 \mathrm{rpm}$ ) and 80 seconds (1000 rpm). The surface pressure results in Fig. 23 was measured at this temperature condition. The temperature fluctuation during this measurement time is $0.05^{\circ} \mathrm{C}$ at $500 \mathrm{rpm}$ and $0.08^{\circ} \mathrm{C}$ at $1000 \mathrm{rpm}$. Using the pressure value error of $0.40 \mathrm{MPa} /{ }^{\circ} \mathrm{C}$ shown in Section 3.2 , the pressure value error due to temperature fluctuation is \pm 0.010 to $0.016 \mathrm{MPa}$ was obtained. This value is $1 / 10$ or less of the surface pressure increase value seen in the section (i ) in Fig. 23, which is clearly slight. Therefore, the influence of temperature on the surface pressure measurement results obtained in this study was judged to be very small.

In addition, instantaneous temperature fluctuations due to asperity contact [20] may occur pressure measurement errors, too. In this study, instantaneous temperature was not measured. However, the minimum oil-film thickness was $1 \mu \mathrm{m}$ or more from the result of the oil-film thickness calculation shown in Fig. 26. Since the oil-film thickness between the piston ring and the cylinder liner are sufficiently thicker than the roughness of the piston ring and the cylinder liner used in the experiments shown in Table 2, it is considered that almost no asperity contact has occurred. Therefore, it was judged that the influence on the pressure measurement value was very small.

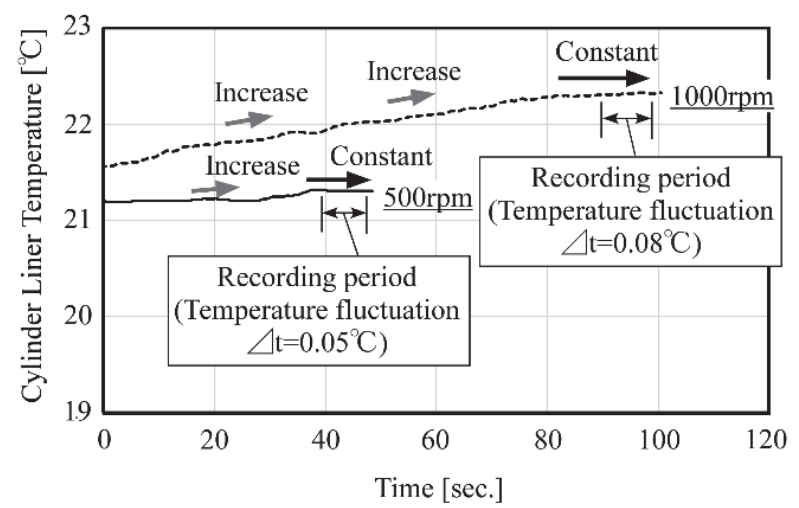

Fig. 25 Measurement result of cylinder wall temperature

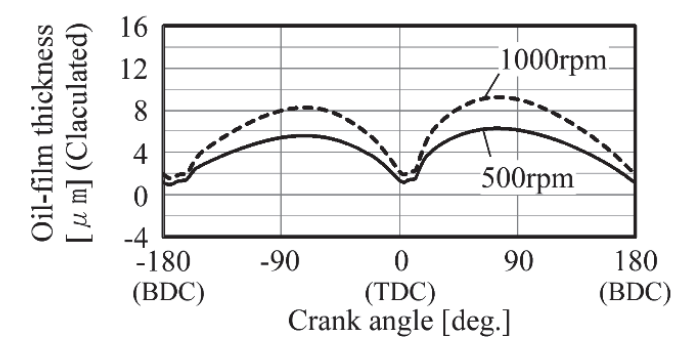

Fig. 26 Theoretical calculation result of oil-film thickness
Table 2 Measurement results of roughness

\begin{tabular}{|c|c|c|}
\hline & $\begin{array}{c}\text { Piston ring } \\
\text { (Sliding surface) }\end{array}$ & $\begin{array}{c}\text { Cylinder liner } \\
\text { (Inner bore) }\end{array}$ \\
\hline$R a[\mu \mathrm{m}]$ & 0.031 & 0.087 \\
\hline$R p k[\mu \mathrm{m}]$ & 0.047 & 0.100 \\
\hline$R v k[\mu \mathrm{m}]$ & 0.079 & 0.348 \\
\hline
\end{tabular}

\section{Conclusions}

In this study, a thin-film pressure sensor for the sliding surface of the piston ring was newly developed to measure the instantaneous pressure distribution when the piston ring slides on the cylinder liner. The measurement results obtained using the reciprocating sliding tester were verified using an theoretical calculation model. To summarize this study;

1. A thin-film pressure sensor having a width of $10 \mu \mathrm{m}$, a length of $0.5 \mathrm{~mm}$ and a thickness of $0.2 \mu \mathrm{m}$ was formed on the piston ring sliding surface by sputtering method. In order to prevent the swelling of the sliding surface shape of the piston ring due to the attachment of the sensor, sputtering was performed over the entire circumference of the piston ring to form a thin-film pressure sensor without changing the basic shape of the piston ring.

2. In order to measure the low surface pressure of $1 \mathrm{MPa}$ or less with high accuracy, the $\mathrm{S} / \mathrm{N}$ ratio was improved 42.9 to $62.9 \mathrm{~dB}(87.1$ to $149.4 \%)$ by modifying the structure of the Wheatstone bridge circuit, filtering reinforcement at preamplifier, gain adjustment at buffer circuit, and coefficient adjustment at A/D converter. Also, the phase delay was improved by up to $13.2 \%$ in the region below $100 \mathrm{kHz}$.

3. In order to clearly determine the zero-point of the measurement value of the thin-film pressure sensor, the piezo quartz sensor was placed to the cylinder bore, and the surface pressure measurement at the moment when the piston ring passes at 53.5 to 58.5 degree crank angle was performed. As a result, a measurement result of $0.34 \mathrm{MPa}$ was obtained. As a result of applying the pressure value of the thin-film pressure sensor at this crank angle to 0.34 $\mathrm{MPa}$, the measurement results by the reciprocating sliding tester obtained maximum values of $0.64 \mathrm{MPa}(500 \mathrm{rpm})$ and $0.52 \mathrm{MPa}(1000 \mathrm{rpm})$ at near TDC (0 degree).

4. By using the mixed lubrication model of Rohde including the Patir \& Cheng model and Greenwood \& Tripp model, the theoretical calculation was performed. The calculation results of continuous contact pressure by theoretical calculation were compared with the surface pressure measurement values by thin-film pressure sensor. As a result, the difference was 3.9 to $6.7 \%$ and good agreement was obtained. In addition, it was found that the difference between the peak position of the calculation and the peak position of the measurement was less than 1 degree ( 0.13 to 0.85 degree).

5. As for the calculation value, the pressure maximum value occurs near 1 degree behind the crank angle 0 degree position, and the pressure peak appears at around 1 degree even in the measurement with the thin-film pressure sensor. This is due to the dynamic effect (squeeze effect) of the oil-film formed between the piston ring and the cylinder bore, and it is considered that the minimum value of the 
oil-film thickness is generated later than the upper and lower dead centers where the speed becomes zero. There has been no verification example in actual measurement of this phenomenon, thin-film pressure sensor verified this phenomenon with high accuracy.

6. Due to temperature changes when the piston ring slides on the cylinder liner, a pressure measurement error may occur depending on the temperature sensitivity of the sensor attached to the piston ring. However, the temperature fluctuation during surface pressure measurement time was $0.05^{\circ} \mathrm{C}$ at $500 \mathrm{rpm}$ and $0.08^{\circ} \mathrm{C}$ at $1000 \mathrm{rpm}$. So, the pressure value error due to temperature fluctuation is \pm 0.010 to 0.016 MPa was obtained. This value is $1 / 10$ or less of the surface pressure increase value seen in the section (i ) in Fig. 23, which is clearly slight. Furthermore, the minimum oil-film thickness was $1 \mu \mathrm{m}$ or more from the result of the oil-film thickness calculation. Since the oil-film thickness between the piston ring and the cylinder liner are sufficiently thicker than the roughness of the piston ring and the cylinder liner used in the experiments, it is considered that almost no asperity contact has occurred. Therefore, it was judged that the influence on the pressure measurement value was very small.

\section{Acknowledgements}

This work was supported by Council for Science, Technology and Innovation (CSTI), Cross-ministerial Strategic Innovation Promotion Program (SIP), "Innovative Combustion Technology" (Funding agency: JST).

\section{References}

[1] Brown, S. R. and Hamilton, G. M., "Negative Pressures under a Lubricated Piston Ring," Journal of Mechanical Engineering Science, 20, 1, 1978, 49-57.

[2] Hitosugi, H., Kanemitsu, K. and Tateishi, Y., "A Study of the Piston Ring Reducing High Contact Pressure at Ring End Gap in Engine Operation," Journal of JSME, 32, 4, 2001, 163-168 (in Japanese).

[3] Cheng, C., Kharazmi, A., Schock, H., Wineland, R. and Brombolich, L., "Three-Dimensional Piston Ring-Cylinder Bore Contact Modeling," ASME Journal of Engineering for Gas Turbines and Power, 137, 11, 2015, 111505.

[4] Rohde, S. M., "A Mixed Friction Model for Dynamically Loaded Contacts with Application to Piston Ring Lubrication," Proc. of the 7th Leeds-Lyon Symp. on Trib., Friction and Traction, Guildford, Butterworth, 1980.

[5] Patir, N. and Cheng, H. S., “An Average Flow Model for Determining Effects of Three-Dimensional Roughness on Partial Hydrodynamic Lubrication," Journal of Lubrication Technology, 100, 1, 1978, 12-17.
[6] Greenwood, J. A. and Tripp, J. H., "The Contact of Two Nominally Flat Rough Surfaces," Proceedings of the Institution of Mechanical Engineers, 185, 1, 1970, 625-633.

[7] Shahmohamadi, H., Mohammadpour, M., Rahmani, R., Rahnejat, H., Garner, C. P. and Howell-Smith, S., "On the Boundary Conditions in Multi-Phase Flow Through the Piston Ring-Cylinder Liner Conjunction," Tribology International, 90, 2015, 164-174.

[8] Rahmani, R., Rahnejat, H., Fitzsimons, B. and Dowson, D., “The Effect of Cylinder Liner Operating Temperature on Frictional Loss and Engine Emissions in Piston Ring Conjunction," Applied Energy, 191, 2017, 568-581.

[9] Baelden, C., "A Multi-Scale Model for Piston Ring Dynamics, Lubrication and Oil Transport in Internal Combustion Engines," Ph.D. Thesis, MIT, 2014.

[10] Yamamoto, K. and Ochiai, M., "Dynamic Contact Analysis of Piston Ring in Distorted Cylinder Bore," Journal of Internal Combustion Engine Symposium, 2016.

[11] Rahmani, R., Shirvani, A. and Shirvani, H., "Optimised Textured Surfaces with Application in Piston-Ring/Cylinder Liner Contact," Tribology and Dynamics of Engine and Powertrain: Fundamentals, Applications and Future Trends, 2010, 470-517.

[12] Vlădescu, S. C., Ciniero, A., Tufail, K., Gangopadhyay, A. and Reddyhoff, T., "Looking into a Laser Textured Piston Ring-Liner Contact," Tribology International, 115, 2017, 140-153.

[13] Owashi, M. and Mihara, Y., "Development of a Measurement Method of Contact Pressure between Gear Teeth Using a ThinFilm Sensor (Measurement of Pressure Distribution by Multi-Point Pressure Sensor with Shared Lead Films)," Journal of JSME (C), 77, 78, 2011, 396-408 (in Japanese).

[14] Miura, K., Owashi, M. and Mihara, Y., "High Durability ThinFilm Pressure Sensor Development for Engine Sliding Surface," The 9th International Conference on Modeling and Diagnostics for Advanced Engine Systems, COMODIA 2017, 2017.

[15] Mochizuki, K., Watanabe, Y., Owashi, M. and Mihara, Y., "A Development of Measurement System for Piston Ring Sliding Surface Pressure," SAE Small Engine Technology Conference \& Exhibition 2018, 18SETC-0084, 2018.

[16] Mochizuki, K., Watanabe, Y., Owashi, M. and Mihara, Y., "Application of Thin-Film Sensing Technique for Surface Pressure Measurement in Piston Ring Sliding Surface," Japan Society of Mechanical Engineering Annual Meeting 2018, J0710203, 2018.

[17] Watanabe, S., "The Strain Gauge and the Application," Nikkan Kogyo Shimbun, Ltd., 1968.

[18] AVL User Handbook, 2011.

[19] Furuhama, S., "A Dynamic Theory of Piston-Ring Lubrication (1st Report, Calculation)," Journal of JSME, 24, 148, 1958, 1032-1039 (in Japanese).

[20] Morris, N., Rahmani, R., Rahnejat, H., King, P. D. and Fitzsimons, B., "Tribology of Piston Compression Ring Conjunction under Transient Thermal Mixed Regime of Lubrication," Tribology International, 59, 2013, 248-258. 